\title{
LAS SOCIEDADES ANTIGUAS DE NICARAGUA. DEL 500 A.C AL 800 D.C.
}

Msc. Sagrario Balladares N

\section{RESUMEN}

Explicar el desarrollo socioeconómico experimentado por las sociedades asentadas en el territorio nicaragüense entre el 500 a.C y el 800 d.C, es el objetivo que persigue el presente documento. Para ello este artículo se ha basado en los datos obtenidos de las investigaciones arqueológicas desarrolladas dentro del territorio nacional. Se destacan las condiciones socioeconómicas y ambientales que permitieron el desarrollo de esas sociedades y su complejidad social, tanto en la ampliación de asentamientos como en la diversificación tecnológica.

\section{Palabras claves}

Arqueología, culturas, periodización, tecnologías e intercambio.

\section{Keywords}

Archaeology, cultures, periodization, technologies and exchange

\section{INTRODUCCIÓN}

Las evidencias arqueológicas encontradas en el actual territorio nicaragüense, demuestran que las sociedades prehispánicas se desarrollaron en diversos momentos cronológicos con una mayor o menor intensidad y de forma diferenciada por todo el territorio. Siempre se ha dicho que el territorio nicaragüense sirvió en el pasado como paso para el tránsito de grupos o culturas que emigraban del norte hacia el sur de América o viceversa, o bien, como parte de los intercambios comerciales que se dieron entre sociedades que habitaban en la región; sin embargo, gran parte de las evidencias arqueológicas documentadas reflejan lo contrario, es decir, muchas de las sociedades asentadas llegaron a establecerse de forma permanente generando su propio desarrollo socioeconómico.

Por lo antes expuesto, serán objeto de un análisis general aquellos sitios $\mathrm{o}$ espacios del actual territorio nacional, donde las sociedades prehispánicas, a nuestro criterio, experimentaron posibles desarrollos locales. El análisis se realiza sobre la materialidad dejada por estos grupos quienes la produjeron a lo largo de este periodo de tiempo; además, se pretende descifrar cómo esas sociedades experimentaron y desarrollaron diversas tecnologías para mejorar sus condiciones de vida. Este aspecto es sumamente importante para conocer el proceso evolutivo de nuestras comunidades

\footnotetext{
* Msc. Leonardo Lechado Ríos, Arqueólogos investigadores del Departamento de Historia UNAN-Managua
} 


\section{LAS EVIDENCIAS ARQUEOLÓGICAS PERIODO (500 A.C. -800 D.C.)}

Los datos arqueológicos existentes para este período se encuentran distribuidos por toda Nicaragua, una realidad fenoménica observable indicadora de la presencia de sociedades en casi todo el territorio nacional; muchas de estas poblaciones practicaban ya -aunque de manera incipiente- la agricultura, actividad que en su desarrollo generó el crecimiento de las poblaciones y la consolidación de grandes asentamientos, ya que hubo un aumento poblacional considerable que complejizó y ocasionó transformaciones en su estructura socio-económica.

Retomaremos la hipótesis que plantea que en el pasado antiguo un grupo conocido como Coribicíes, arribó al territorio nacional alrededor del siglo IV antes de Cristo, estableciéndose en el Pacífico nicaragüense. Estos permanecieron allí hasta el momento en que ocurrieron las primeras migraciones mesoamericanas hacia el actual territorio nacional en el siglo VIII ${ }^{1}$ obligándolos a desplazarse a diferentes regiones del país.

Por la escasez de evidencias materiales vinculante con el análisis más completo de estos grupos y por la poca variabilidad tecnológica documentada, al parecer en todosu devenirhistóriconoexperimentaron grandes cambios socio-económicos, al menos durante este periodo; sin embargo, todo indica que la producción cerámica, posiblemente tenía vínculos directos con actividades agrícolas.

Al respecto y refiriéndose a Salgado,(1996), J. Zambrana,2002, señala que "en el Periodo Bagaces (300-500 d.C.), se observa un incremento de la población basado en el mayor tamaño que exhiben los sitios arqueológicos. Para el período Tempisque-Orosí, la población del área en estudio se estima en 1200 y para Bagaces en 3150. Se observa a partir de éste período una clara jerarquía de asentamientos. Es decir, en Granada comienza a desarrollarse una organización socio-politica de carácter territorial que puede definirse como cacicazgo. Surge, en este período, evidencia de contacto con otras sociedades al norte y al sur de nuestras actuales fronteras, basados en la identificación de cerámica hondureña, salvadoreña y costarricense; además, de artefactos líticos procedentes del norte como la obsidiana, y el jade, posiblemente procedente de la actual Costa Rica, y el surgimiento de élites que controlaban el intercambio, surgiendo aparentemente centros regionales como Ayala en Granada y Playas Verdes en Masaya".

En la región norte del país la evidencia arqueológica $^{2}$, refleja que en el actual territorio que conforma los departamentos de Estelí, Madriz, Nueva Segovia, Jinotega y Matagalpa $^{3}$ existían poblaciones con dinámicas socio-económica muy bien consolidadas, sin lograr establecer alguna filiación cultural. Por ejemplo, en el mapa (imagen 2) se proyecta una posible ruta seguida por los antiguos habitantes de Nicaragua, para entablar relaciones

1 Las migraciones mesoamericanas hacia el actual territorio nicaragüense, se produjeron entre los siglos VIII y XV d. C

2 San Antonio Negativo, Las Segovias Naranja, Condega engobe Rojo, Rocinante Común, Cacaulí Rojo sobre Naranja, Las Tapias Tricromo, Güiligüisca Inciso, Ulúa polícromo, Tenampúa polícromo. La mayoría de ellas ubicadas tipológicamente entre el 300 y 1000 dc. También se retoma el Usulután Negativo y Bocana Inciso porque son tradiciones cerámicas que inician aproximadamente en el 500 a.C y se extiende hasta el 300 d.C.

3 Entre el periodo que comprende los años 2006 y 2010, se realizaron en el departamento de Matagalpa y los municipios de Pantasma y Jinotega (estos dos últimos pertenecientes al departamento de Jinotega) investigaciones arqueológicas que perseguían la documentación del potencial arqueológico de ese departamento. Se identificaron más de 200 sitios de interés arqueológico. 
socioeconómicas entre las poblaciones del Pacífico, Centro y Caribe nicaragüense. Esta ruta fue elaborada en base a un análisis comparativo de las tecnologías cerámicas encontradas en los departamentos arriba señalados, obteniendo como resultado que presentan los mismos principios tecnológicos para su producción con algunas leves diferencias (imagen 1 ). Unas de las características importantes que presentan estas evidencias materiales son, por ejemplo, la técnica en la elaboración y la decoración de los distintos tipos cerámicos encontrados.
La presencia de esta tecnología cerámica denota claramente que la región norte de Nicaragua, estuvo poblada durante el período comprendido entre 300 al 1000 d.C, y tal parece que durante ese período, la región actualmente denominada $\mathrm{La}$ Segovia, formó parte de una red de intercambio bien estructurada con la actual Granada y otras poblaciones localizadas en la cuenca del lago de Managua. La presencia de este tipo cerámico en estas áreas, así lo indican. Por otra parte, la existencia de este mismo tipo de materiales en Acahualinca junto a materiales del período Bagaces (300-800 d.C), refuerzan esta hipótesis.
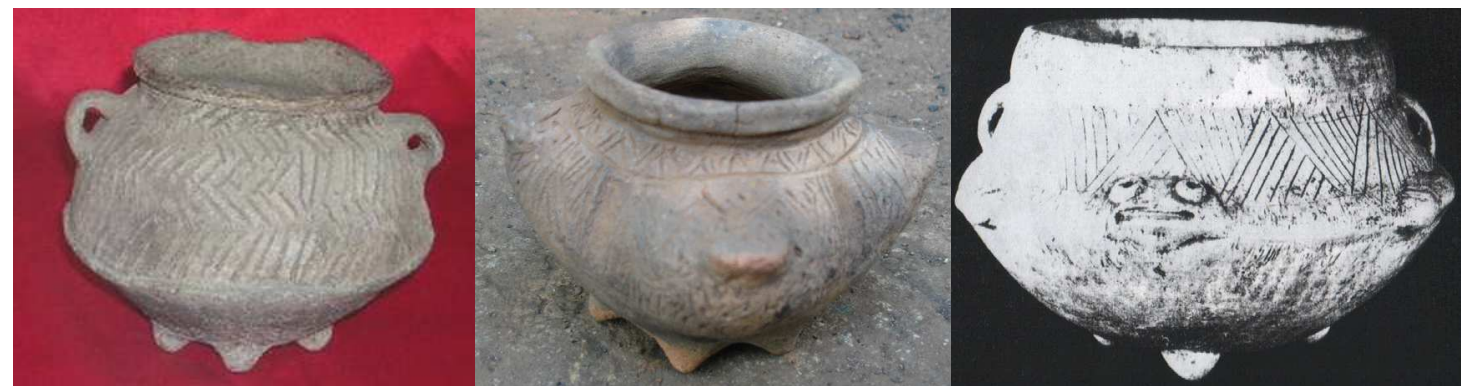

Imagen 1. Similitudes tecnológicas en la producción cerámica. Izquierda: pieza localizada en el museo de Condega, encontrada en la zona (Fuente Zambrana y Gámez, 2006; 10); al centro, pieza recuperada en el municipio de Kukra Hill, RAAS. Fuente: CADI; derecha: pieza de tipo Usulután (-200 a +550dc), referida por Claude F. Baudez cuyo origen remite al municipio de León (Baudez, 1976:41 y 243). El principio de decoración es similar en todas, (forma, soportes, incisos y apliques).

Para la región del Caribe, desde el año 1998 se han realizado esfuerzos con el propósito de comprender los desarrollos socioeconómicos experimentados por las poblaciones antiguas asentadas en la Costa Caribe. Las investigaciones arqueológicas ${ }^{5}$ permitieron localizar abundantes sitios prehispánicos, muchos de ellos sencillos pero en su gran mayoría conformados por amplios asentamientos humanos, reflejando una complejidad social de esos antiguos habitantes.
En este período, la mayor cantidad de sitios documentados corresponden a sitios arqueológicos de tipo concheros, localizados en las antiguas zonas costeras, específicamente en Laguna de Perlas y Bahía de Bluefields, reflejando de una $\mathrm{u}$ otra manera, modos de vida humano especializados en la explotación marina complementando con actividades de caza y de recolección; pero igualmente se han documentado otro tipo de sitios conformados por estructuras monticulares, 5 Los estudios en la costa Caribe han permitido la documentación de más de 85 sitios concheros, reflejando el nivel de organización y
poblamiento de la zona, así como también se han realizado 18 fechamientos radiocarbónicos que reflejan ocupaciones que oscilan entre el 1400 calAC y el 950 calDC. 


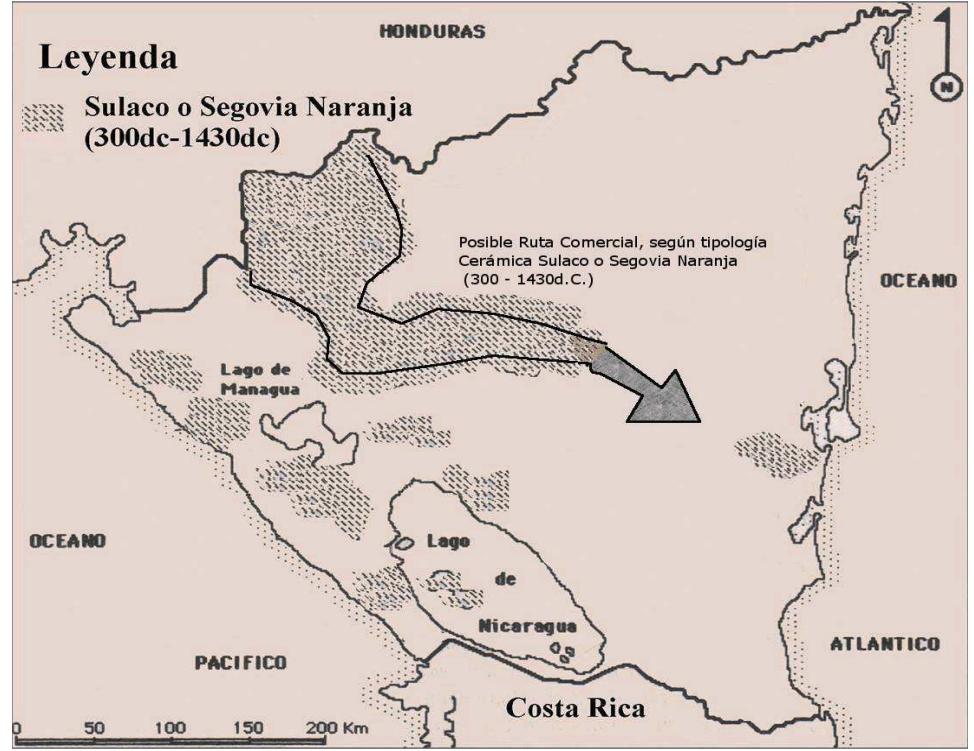

Imagen 2. Distribución de Cerámica Tipo Sulaco o Segovia Naranja (300-1430 d.C). Nótese posible ruta comercial para estos periodos. Elaborado sobre la base de investigaciones arqueológicas. Fuente CADI. 2011.

terrazas, predominando en ellos materialidad indicadora del desarrollo de actividades de caza, recolección de recursos terrestres y de actividades vinculadas al quehacer agrícola. Ejemplo, los sitios arqueológicos de EL Cascal en Kukra Hill, y Tecaniste en Nueva Guinea, ambos en la RAAS, en Rosita y Waspan (RAAN). Además se han documentado petroglifos y áreas de talla de artefactos líticos. Lo cierto es que la mayor complejidad socioeconómica de la región se dio entre el 900 a.C y el 900 d.C. Las imágenes reflejan posibles influencias culturales entre sociedades establecidas en la Costa Caribe y la región Central-Norte de Nicaragua.

Es posible identificar intercambios en las diversas regiones que conforman nuestro actual territorio, por ejemplo en el sitio
Sitetaia, donde se encontró cerámica de tipo Chombo (200 a. C y 400 d. C) cuyo origen se atribuye a la sub área cultural de la Gran de Nicoya, (Magnus, 1974). La presencia de cerámica policromada de tipo Luna en el municipio de Nueva Guinea, sugiere posibles contactos con poblaciones del Pacífico nicaragüense.

Las comunidades indígenas asentadas sobre las riberas de de los ríos como: el San Juan, el Mico, el Sarapiquí, etc, dominaban las condiciones de la selva tropical húmeda, hecho que permitió que tuvieran control de varias rutas de intercambio, no sólo al interior del actual territorio nacional, sino con otras comunidades localizadas tanto al norte ${ }^{6}$, como al sur ejemplo, con comunidades asentadas en las riberas del río Frío, en el actual territorio costarricense.

Referente a los intercambios regionales, Braswell y Salgado, 1996, expresan que la mayoría de artefactos de vidrio volcánico recuperados sobre todo en la Gran Nicoya, fueron importados desde Honduras y/o Guatemala ${ }^{7}$ ya sea en forma de materia prima o como instrumentos acabados, y que la mayoría de los artefactos en obsidiana encontrados en el período Bagaces, provenían del noreste y se difundía lentamente al sureste de Centroamérica.

6 En el sitio La Granja, Boaco, se obtuvieron fragmentos de cerámica de tipo Segovia Naranja, típica de la región norte o Segovia del país; sin embargo, los fragmentos fueron encontrados en los dos primeros niveles del sondeo, lo que probablemente estaría indicando el establecimiento de relaciones tardías entre ambos grupos. Algunos autores, también relacionan la presencia de obsidiana como indicador de relaciones con Honduras.

7 Braswell, Salgado, expresan que grupos de Nicaragua explotaban varias fuentes de obsidiana en Mesoamérica, especialmente los yacimientos del Güinope y la Esperanza en Honduras, y del altiplano Guatemalteco Ixtepeque y el Chayal, esta relación se estableció gracias a métodos de análisis petrográfico químico y visual (activaciones de neutrones ANN). 


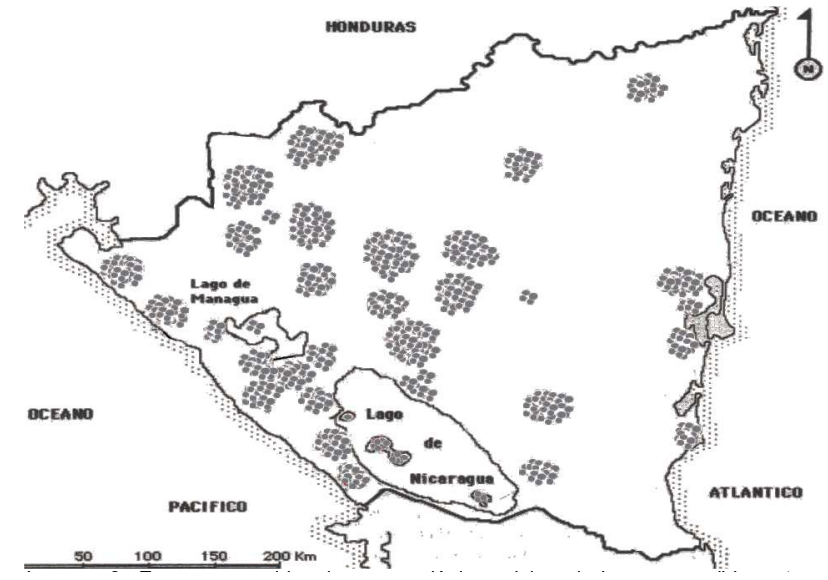

Imagen 3. Zonas con evidencias arqueológicas del periodo comprendido entre 500 ac-800dc. Mapa elaborado sobre base de investigaciones recientes. Fuente CADI. 2011

Implícitamente se está demostrando que existía una distancia considerable para obtener la materia prima, o bien el mismo intercambio facilitaba esto. También se ha dicho que existía intercambio con otros grupos dentro del territorio nacional, ejemplo, en el sitio los Placeres en Managua, la presencia de pedernal y jaspe se asocia a intercambio con la región de Chontales. (Lange y Sheets, 1983).

Con respecto al Pacífico nicaragüense, la evidencia arqueológica correspondiente a este periodo se concentra mayormente en los departamentos de Managua, Carazo y Granada, sobretodo en la parte baja del primer milenio después de Cristo. Estas evidencias se reducen considerablemente en los departamentos de Rivas, Masaya, León y Chinandega. Por ejemplo, el sitio El Apante con una antigüedad de 800 años aproximadamente y localizado en el municipio de Telica del departamento de León ${ }^{8}$, se excavó por primera vez una estructura arquitectónica precolombina, documentándose paredes elaboradas en piedra y barro construidas sobre una terraza artificial, también se empleó el bahareque como parte de ese sistema constructivo.

Lo cierto es que en esta región, existieron muchas poblaciones humanas que desarrollaron sus propios sistemas de organización socio-económicos, donde los intercambios comerciales o contactos con otras regiones fueron sumamente importantes.

Braswell, G. y Salgado, S. 1996, realizaron estudios sobre instrumentos líticos en los sitios Guiligüisca y Cacaolí, ubicados en Madríz y la parte Central - Norte de Nicaragua; también en el sitio Ayala (Fletcher, et al. 1994, cit. Braswell) ubicado cerca de la ciudad de Granada. En estos sitios se aplicaron diversos análisis petrográficos y químicos para conocer las fuentes de aprovisionamiento de las materias primas para la elaboración de los artefactos líticos. Los resultados permitieron concluir que la mayoría de artefactos de vidrio volcánicos recuperados en estos sitios, sobre todo en la Gran Nicoya, fueron importados desde Honduras y/o Guatemala, ya sea en forma de materia prima o como instrumentos acabado, y que la mayoría de los artefactos en obsidiana encontrados en Nicoya durante el periodo Bagaces, provenían del noreste y se difundían lentamente al sureste de Centroamérica (Braswell, G. y Salgado, S. 1996).

Lo interesante de este periodo es que existieron aldeas, así como pequeños y amplios poblados distribuidos por todas las regiones del país y no todos pertenecían a una misma cultura -al menos eso deja entrever la materialidad-; las redes de intercambios socio-económicos

8 Este proyecto de investigaciones arqueológicas en León se ejecutó de 1997 y 1998 por la UNAN, Managua y la Universidad Autónoma de Barcelona (UAB). 
se encuentran bien consolidadas en todo el territorio nacional e incluso con otros grupos asentados en la región centroamericana (Guatemala, Honduras, Costa Rica y El Salvador), muchas de estas redes tuvieron sus orígenes en la etapa final del periodo anterior y tuvieron su máxima expansión hasta mediados del primer milenio (después de Cristo).

Esa interacción regional se refleja, no solo en la materialidad, sino también, en el intercambio de expresiones culturales y la apropiación de ideas, las que son posibles observar en la combinación de estilos e iconografía cerámica y en el arte rupestre.

Es importante destacar que antes de la llegada de los grupos mesoamericanos, el territorio se encontraba ampliamente ocupado; se estaban terminando de consolidar muchos poblados, pero la llegada de estas nuevas culturas provenientes del norte de la región CA, marcaron significativamente ese desarrollo paulatino que venían experimentando las sociedades desde muchos siglos atrás, obligándolas, a -algunas- desplazarse a otras regiones del territorio nacional, $\mathrm{y}$ otras, a mezclarse con las nuevas recién llegadas.

La llegada de los mesoamericanos significó una transculturación generándose una mezcla cultural en todas las actividades $\mathrm{y}$ las subsiguientes. Este fenómeno migratorio debe relacionarse, no solo con los conflictos bélicos, sino con la crisis económica que estaban viviendo los grupos asentados en el norte de la región centroamericana, crisis que se reflejó en conflictos entre grupos y en la búsqueda de nuevos recursos, en la ampliación de rutas comerciales o nuevas rutas de intercambio que proporcionaran a esos pueblos un respiro; probablemente, estas fueron también causas que provocaron las migraciones mesoamericanas. Se requieren mayores análisis de estos fenómenos para profundizar en este campo.

\section{CONSIDERACIONES FINALES}

Nicaragua ha sido un territorio de convergencia e influencia de diversas culturas, y por su posición geográfica un corredor natural de tránsito y de confluencia de culturas provenientes tanto del norte como del sur de la región centroamericana, causa de la presencia de una gran variedad de cultura material a lo largo y ancho de todo el territorio nacional.

A pesar que la mayor cantidad de estudios arqueológicos están dirigidos a épocas más recientes, en este estudio se ha logrado definir hipotéticamente, algunos aspectos de la evolución social del modo de vida de las sociedades asentadas en las distintas regiones del país desde épocas antiguas hasta la llegada de los mesoamericanos, combinando en ocasiones estos datos con los proporcionados por la lingüística.

Desde la Arqueología se puede afirmar que el territorio nacional estuvo densamente poblado en el período estudiado, formando parte de una red de intercambio con pueblos de Honduras, El Salvador y pueblos al interior del país; por ejemplo, existió toda una red de intercambio muy bien estructurada entre las poblaciones localizadas en la cuenca del lago de Xolotlán o Managua y el Cocibolca o Nicaragua, incluyendo los actuales departamentos de Chontales, Río San Juan y la Región Autónoma del Atlántico Sur, RAAS; la presencia de cerámica con engobe Naranja de tipo Sulaco o Segovia Naranja en estas áreas, así lo indican. Esta red se vio interrumpida brevemente a causa de la llegada de los primeros grupos mesoamericanos al Pacífico nicaragüense en el siglo VIII. 
Las relaciones sociales y económicas que se generaron entre esta gama de culturas, permitieron las apropiaciones, adaptaciones y adecuaciones tecnológicas de los grupos, haciendo más difícil la delimitación de las áreas de influencias de uno u otro grupo humano a partir tan sólo de la materialidad.

\section{BIBLIOGRAFÍA}

Arellano Jorge E. (2001): "Chorotegas y Nicaraguas: Cultura material, sociedad y cosmovisión. Culturas indígenas y Pasado Colonial de Nicaragua; Boletín número 112, editorial Nacional, año 2001, Pág. 22 $-31)$

Arellano Jorge Eduardo (Editor) 1993. "Ometepe y el Sur de Centroamérica". En 30 Años de Arqueología en Nicaragua. . Museo Histórico de Suecia-Museo Nacional de Nicaragua,

Arellano, Jorge Eduardo. 1998. “El mundo pre-hispánico de Nicaragua". En Culturas Indígenas de Nicaragua Tomo I. Editorial Hispamer, Managua.

Arellano, Jorge Eduardo. 1997.Historia Básica de Nicaragua, Volumen I. Fondo Editorial CIRA. 1ra. y 2da. Edición, Nicaragua 1993 y 1997.

Balladares, S. et al. 2006. “Una Aproximación A la Historia Antigua de la Región de Las Segovias" en, La Región Segoviana, Evolución Histórica de Nueva Segovia, Madriz y Estelí. Edit. Acento. UNAN-Managua. Pp.33-52.

Balladares, S. y Lechado, L. 2005. "Historia Antigua de la Región del Pacífico, Centro y Sur de Nicaragua" en, El Oriente y
Mediodía de Nicaragua aportaciones al estudio de su historia. Edit. Acento. UNAN-Managua. Pp. 15-28.

Balladares, S. y Lechado, L. 2005. "Historia Antigua de la Región de Occidente de Nicaragua" en, Tierra Ardiente, El Occidente de Nicaragua a Través de $\mathrm{Su}$ Historia. Edit. Acento. UNANManagua. Pp.9-29

Balladares, S. y Lechado, L. 2005. “Una Aproximación a la Historia Antigua de la Región de Matagalpa y Jinotega" en, Región Norte Central de Nicaragua. Matagalpa y Jinotega a través de su historia. Edit. Acento. UNANManagua. Pp.19-31.

Balladares, S. y Lechado, L. 2007. "Aproximación a la Historia Antigua de la región del Caribe Nicaragüense" en, Historia de la Costa del Caribe de Nicaragua. Un enfoque Regional. Edit. Acento. UNAN-Managua. Pp. 37-55.

Balladares, S. y Lechado, L. 2009. " $\mathrm{La}$ Historia Antigua del Departamento de Managua. Una breve interpretación de la evidencia arqueológica" en, Managua y Su Historia. Un Enfoque regional. Edit. Acento. UNAN-Managua. Pp.40-63.

Balladares, S. y Lechado, L. 2009. “Los Grupos Humanos Precolombinos de las regiones Norte, Centro y Pacífico de Nicaragua" en, Nuestras Comunidades Una Mirada Histórica de los Pueblos Indígenas del Pacífico, Centro y Norte de Nicaragua. Edit. UNAN-Managua. Pp-15-50.

Chapman, Anne. 1974. "Los Nicarao y los Chorotega", según las Fuentes históricas. Universidad de Costa Rica, Serie Historia y Geografía No. 4. 
Esgueva G. A. 1996. La Mesoamérica Nicaragüense. UCA, Managua.

Espinoza, E.; Fletcher, L.; Salgado, R. 1999. "Arqueología de las Segovias: Una Secuencia Cultural Preliminar". Instituto Nicaragüense de CulturaOrganización de los Estados Americanos-Museo Nacional de Nicaragua. Editor Mario Molina C, Nicaragua.

Espinoza, González y Rigat. 1992-93. Gran Nicoya y la cuenca del Lago de Managua. VINCULOS, 18-19, 157172. Museo Nacional de Costa Rica.

Fonseca, O y Cooke, R. 1993. "El sur de América Central: contribución al estudio de la región histórica Chibcha". Historia General de Centroamérica. T. I, Cap. 4. Edición R. Carmack.

Gassiot B. Ermengol y Palomar P. Beatriz. 1998. Primer informe preliminar Arquelogía en Miraflor, Estelí, Nicaragua. UNAN, Managua.

Gassiot, E. y Palomar, P. 1999. "Informe de la Campaña de Prospecciones en Laguna de Perlas, Kukra Hill y Bluefields". No publicada. UNANManagua.

Graham, 1993. Reinterpreting Prehistory of Central America. University of Colorado, Boulder.

Haberland, W. 1992. "The Culture History of Ometepe, Island: Preliminary Sketch (Survey and Excavations, 1962-1963)". En Archaeology of Pacific Nicaragua. Editor Frederick W. Lange. University of New Mexico Press.

Healy, P. 1980. "Archaeology of the Rivas Region Nicaragua". Waterloo,
Ontario. Wilfried Laurier University Press.

Hno. Hildeberto María. 1965. Estas piedras hablan. Presidencia República.

Ibarra R, Eugenia. 1993. Los Matagalpas a principios del siglo XVI. Aproximación a las relaciones interétnicas en Nicaragua (15221581). Vínculos, Vol 18 y 19, Nº 1 y 2.

Incer, Jaime. 1985. “Toponimias indígenas de Nicaragua". Asociación Libro Libre, San José.

Khüll, Eddy. 2002. Matagalpa y sus gentes.

Kinloch, T. Frances. 2006. Historia de Nicaragua 2da. Edición Instituto de Historia de la Universidad Centroamericana. Managua Nicaragua.

Lange Frederick . et al. The archaeology of Pacific Nicaragua. New México.

Lange W. F. 1984. "The Greater Nicoya Archaeological Subarea". En The Archeology of Lower Central America. Editores Frederick W. Lange y Doris Stone. By The School of American Research. University of New Mexico Press.

Lange, W. F., y Stone D. 1984. "The Archeology of Lower Central América". (Introducción). Editores Frederick W. Lange y Doris Stone. By the School of American Research. University of New Mexico Press.

Lechado Ríos, Leonardo. (inédito). 2001. Propuesta de una metodología de análisis para el material arqueológico lítico. Caso de estudio Instituto Miraflor. CADI, UNAN, Managua.

Lechado L, Clemente I, Gassiot E y Verdum E. 2004. Memorias RAAS. 2004. 


\section{CADI-UNAN-Managua.}

Lohhrop, Samuel. 1998. "Las culturas indígenas pre-hispánicas de Nicaragua y Costa Rica". En: Culturas indígenas de Nicaragua, Tomo I. Editorial Hispamer, Managua, 1998.

Magnus, R. 1974. "The Prehistory of the Miskito Coast of Nicaragua: A Study in Cultural Relationships". (Tésis de Doctorado). Yale University. No publicada.

Matilló Vila, J. 1977. “Acahualinca en el Panorama Arqueológico de Nicaragua". Editorial Unión. Managua. Nicaragua.

Moscoso, Francisco. 1991. "Los cacicazgos de Nicaragua antigua". Universidad de Puerto Rico, San Juan.

Newson, Linda A. 1987. Indian survival in Colonial Nicaragua. The University of Oklahoma Press, Norman and London.

Niemel, Karen. 2000. La migración posclásica de los Chorotega y Nicarao al pacífico de Nicaragua. Nuevos datos del Istmo de Rivas. XIV simposio de investigaciones arqueológicas en Guatemala. Museo Nacional de Arqueología y Etnología. Guatemala.

Palomar, et al. 2000. ¿Poder para o poder sobre? Arquitectura y Montículos prehispánicos en Nicaragua. Ponencia presentada en el 50o Congreso Internacional de americanistas. Varsovia, Julio 2000.

Revista de Antropología. 1994. “El Concepto de Area de Tradición Chibchoide y su Pertinencia para Entender Gran Nicoya". En: Vínculos No. 18 (1-2). Museo Nacional de
Costa Rica.

Rodríguez, Ramona; et al. 2006. Biogeografía de la Segovia. En La Región Segoviana. Evolución Histórica de Nueva Segovia, Madriz y Estelí. ACENTO S.A. UNAN, Managua.

Romero, Germán. 1996. Historia de la Costa Atlántica. CIDCA-UCA, Nicaragua.

Salgado, S. 1996. (inédito). “Social Change in Granada, a Region Nicaragua 1500 a.C-1500 d.C". Tésis de Doctorado.

Smutko, Gregorio. 1985. La mosquitia. Historia y cultura de la Costa Atlántica. Editorial La Ocarina, Nicaragua.

Snarkis, M. e Ibarra, E. 1985. Comentarios sobre el intercambio entre la Gran Nicoya, la Vertiente Atlántica y el Valle Central de Costa Rica en Períodos Precolombinos e Históricos. VINCULOS. Vol 11, N 1-2- Museo Nacional de Costa Rica.

Van Broekhoven, Laura. 2002. Conquistando lo invencible. Fuentes históricas sobre las culturas indígenas de la región central de Nicaragua. CNWS Publications, Leiden University, The Netherlands.

Vínculos. 1990. Revista de Antropología del Museo Nacional de Costa Rica. Volumen 13, Número 1-2. 327 pág.

Zambrana F, Jorge. 2002. Los primeros grupos humanos en Nicaragua. En Historia de Nicaragua. Texto Básico. INIES.UNAN, Managua. 